\title{
FACT - Status and Experience from Three Years Operation of the First SiPM Camera
}

\author{
A. Biland $^{* a}$, M. L. Ahnen ${ }^{a}$, M. Balbo ${ }^{b}$, M. Bergmann ${ }^{c}$, T. Bretz ${ }^{a, 1}$, K. A. Brügge ${ }^{d}$,

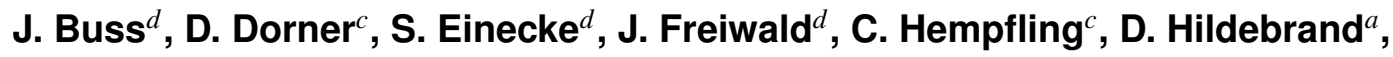 \\ G. Hughes ${ }^{a}$, W. Lustermann ${ }^{a}$, K. Mannheim ${ }^{c}$, K. Meier ${ }^{c}$, S. Müller ${ }^{a}$, D. Neise ${ }^{a}$, \\ A. Neronov ${ }^{b}$, M. Nöthe ${ }^{d}$, A.-K. Overkemping ${ }^{d}$, A. Paravac ${ }^{c}$, F. Pauss ${ }^{a}$, W. Rhode ${ }^{d}$, \\ F. Temme ${ }^{d}$, J. Thaele ${ }^{d}$, S. Toscano ${ }^{b}$, P. Vogler ${ }^{a}, \mathbf{R}$. Walter ${ }^{b}$, and A. Wilbert ${ }^{c}$ \\ ${ }^{a}$ ETH Zurich, Institute for Particle Physics \\ Otto-Stern-Weg 5, 8093 Zurich, Switzerland \\ ${ }^{b}$ University of Geneva, ISDC Data Center for Astrophysics \\ Chemin d'Ecogia 16, 1290 Versoix, Switzerland \\ ${ }^{c}$ Universität Würzburg, Institute for Theoretical Physics and Astrophysics \\ Emil-Fischer-Str. 31, 97074 Würzburg, Germany \\ ${ }^{d}$ TU Dortmund, Experimental Physics 5 \\ Otto-Hahn-Str. 4, 44221 Dortmund, Germany \\ 1 also at RWTH Aachen \\ E-mail: bilandephys.ethz.ch
}

The First G-APD Cherenkov Telescope (FACT) is pioneering the usage of solid state photosensors (G-APD, also known as SiPM). The 1440 pixel camera is installed in a $9.5 \mathrm{~m}^{2}$ refurbished HEGRA telescope on the Canary Island La Palma.

Physics data-taking with FACT started in October 2011, a few hours after installation of the camera. Since Summer 2012, FACT is operated remotely without the need of a data-taking crew on site. During more than three years of operation of FACT, G-APDs have proven to be very reliable. Despite operating them regularly also under very strong moonlight conditions, the GAPDs show no change in their properties or any indication for aging. This allows FACT to have a successful monitoring program of the brightest $\mathrm{TeV}$ blazars in the Northern hemisphere and several flare-alerts have been sent to the community.

This proceeding summarizes the history and status of FACT as well as reporting the lessons learned about the usage of SiPM in a Cherenkov telescope from the construction and operation of FACT.

The 34th International Cosmic Ray Conference,

30 July- 6 August, 2015

The Hague, The Netherlands

\footnotetext{
*Speaker.
} 


\section{Introduction}

The First G-APD Cherenkov Telescope (FACT) $[1,2]$ is located at the Roque de los Muchachos observatory on the Canary Island La Palma at an altitude of $2200 \mathrm{~m}$ a.s.l. (fig.1). The main reason for the construction was to investigate whether novel solid state photosensors are a viable alternative for future cameras of Cherenkov telescopes. The FACT camera has a field of view of $\approx 4.5^{\circ}$ and consists of 1440 pixel with individual readout taking two gigasamples per second. It is mounted on a refurbished HEGRA telescope with a mirror area $\approx 9.5 \mathrm{~m}^{2}$. The system is sensitive enough for monitoring bright $\mathrm{TeV}$ sources [3] and can free the larger IACTs from this time consuming task. In addition, FACT shows an excellent longterm stability, ideal for monitoring tasks. The (nearly) real-time analysis of the observations are publicly available at http://www.factproject.org/monitoring/. This quick-lookanalysis is based on [4] and described in [5]; a second, fully independent analysis chain [6] is under development.

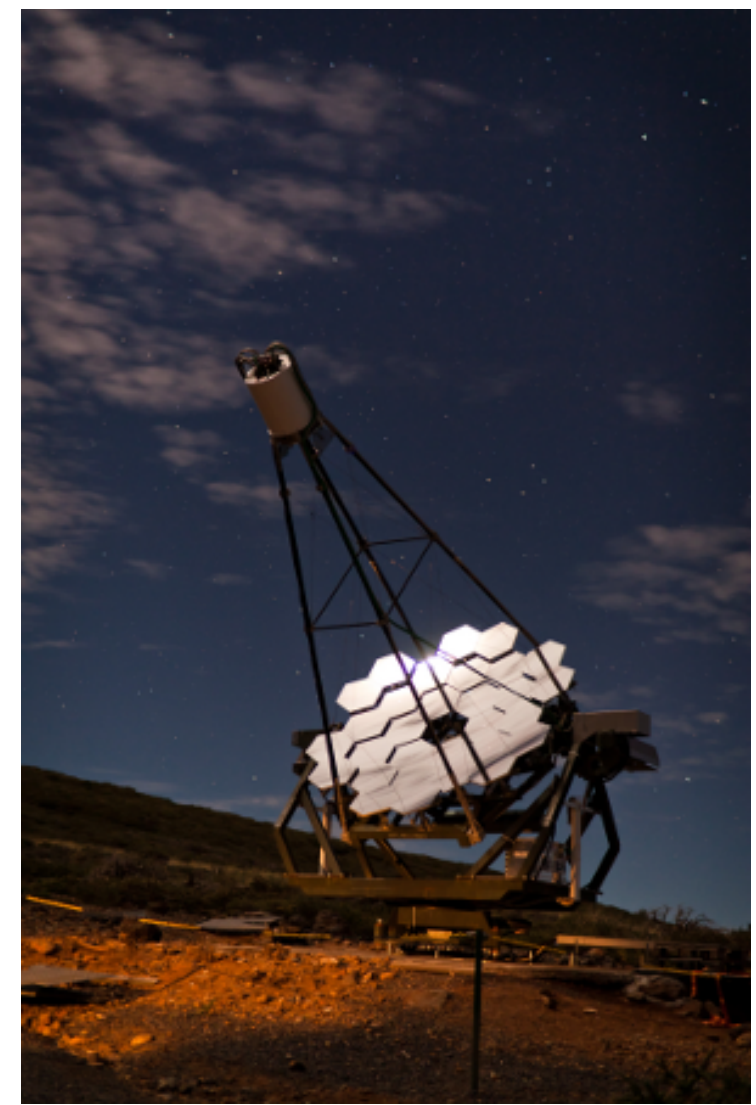

Figure 1: The First G-APD Cherenkov Telescope at La Palma (picture by J.L. Lemus).

\section{History}

Imaging Atmospheric Cherenkov Telescopes (IACT) measure the dim, short flashes of Cherenkov light emitted by secondary particles in extensive air showers in the atmosphere. These showers are induced by very high energy photons and cosmic ray particles. To be able to register these Cherenkov flashes, cameras with single-photon sensitivity, able to operate at several hundreds to thousands of megasamples per second, are necessary.

So far, all IACTs use cameras based on Photo-Multiplier Tubes (PMT). A few years ago, the first Avalanche Photo Diodes operated in Geiger-mode (G-APD aka SiPM) [7] with a performance comparable to PMTs became commercially available. This raised the question whether G-APDs might be a viable alternative for next generation IACTs. First successful tests to measure Cherenkov flashes were reported in [8]. Despite this first successes, it was widely assumed these novel sensors not yet to be ready for longterm usage in the very harsh environment intrinsic to the operation of IACTs, and G-APD based research was excluded from the ongoing CTA design study [9]. Some people were more optimistic, and groups from ETH Zurich and the Universities Dortmund, Geneva and Würzburg formed the FACT collaboration to build and operate a camera, 
installed in a refurbished mount of an old HEGRA telescope. The first step was to build a module consisting of 144 G-APDs. Four G-APDs were grouped together to form one pixel, resulting in 36 pixels with a field of view $\approx 0.2^{\circ}$ each. The original plan was first to test this Module 0 extensively in the laboratory. In a second stage, several such modules should form an $\approx 3^{\circ}$ camera to be tested in the HEGRA mount at La Palma. Based on experience of operating this prototype system for several months, more modules were planned for a final camera of $\approx 5^{\circ}[10]$.

In summer 2009, Module 0 was operated on the roof of ETH in Zurich and despite very high ambient light immediately recorded the first self-triggered Cherenkov images [11]. Despite this success, there was the problem that available electronics was too bulky and/or expensive. Due to the unexpectedly few problems found in the construction and operation of Module 0, the collaboration became more ambitious and decided to go directly for the final camera. New readout electronics based on the DRS-4 analog ring sampler chip [12] was developed to be fully integrated in the camera body and being able to read out 1440 individual pixels [14]. A pixel is formed by a single G-APD chip (Hamamatsu MPPC S10362-33-50C [13]) with an area of $3 \times 3 \mathrm{~mm}^{2}$ containing 3600 individual cells. Each chip is coupled to a hexagonal-to-square solid light concentrator with a concentration factor of $\approx 10$, and has a field of view of $\approx 0.1^{\circ}$ per pixel.

This development was completed within two years and the camera installed at La Palma in October 2011. A detailed description of the system can be found in [1]. Within few hours, the first Cherenkov showers were recorded, and first scientific results were presented in July 2012 [15]. While it was foreseen to take the camera back to the laboratory after few months of operation to modify it according to the lessons learned in the field, the system has performed so well since the first night that these plans were cancelled. Since installation, FACT was operated during more than 1000 nights so far without any measurable problem related to the photo sensors.

In the first year of operation, data taking was affected by many tests of the system, lack of funding to maintain continuous shift coverage on La Palma, and time necessary to develop and debug the software for automatic processing. Since autumn 2012, automatic operation is normally controlled from remote without the need of data taking personnel on site (in case of emergencies, the crew of the nearby MAGIC telescopes could be asked for help). Since then, when weather conditions permit, FACT has reached a data taking efficiency of $>90 \%$, defining $100 \%$ as the time between astronomical twilights (fig.2). Most of the remaining time is spent to reposition the telescope as well as taking trigger ratescans and other tests.

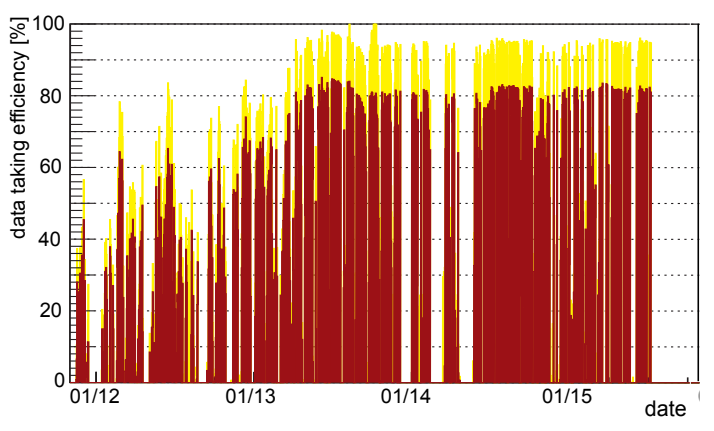

Figure 2: Data taking efficiency: red is the time spent for standard data runs, while yellow are special data runs to monitor the stability of the system. The missing time to $100 \%$ (defined between astronomical twilights) includes time to reposition the telescope and to take additional tests. Large gaps in data taking are due to exceptionally bad weather periods or hardware problems (mainly with commercial components). 


\section{Sum-Trigger}

To reach a low energy threshold, FACT uses a sum-trigger [16]. To simplify electronics and cabling, it was decided to use non-overlapping but rather small trigger patches, i.e. showers large enough to get reasonable background suppression will be larger than one patch. A natural choice for hexagonal pixels would have been to use patches of seven pixels. But since there is one readout chip for nine pixels, using the same multiplicity was the easiest solution (fig. 3). A major obstacle for sum-triggers is the danger that a single pixel containing a very large signal (e.g. due to afterpulses in the case of PMTs) is sufficient to trigger the full camera. While afterpulsing in G-APDs is not a similar problem as in PMTs, one has to be careful about optical crosstalk within a chip that

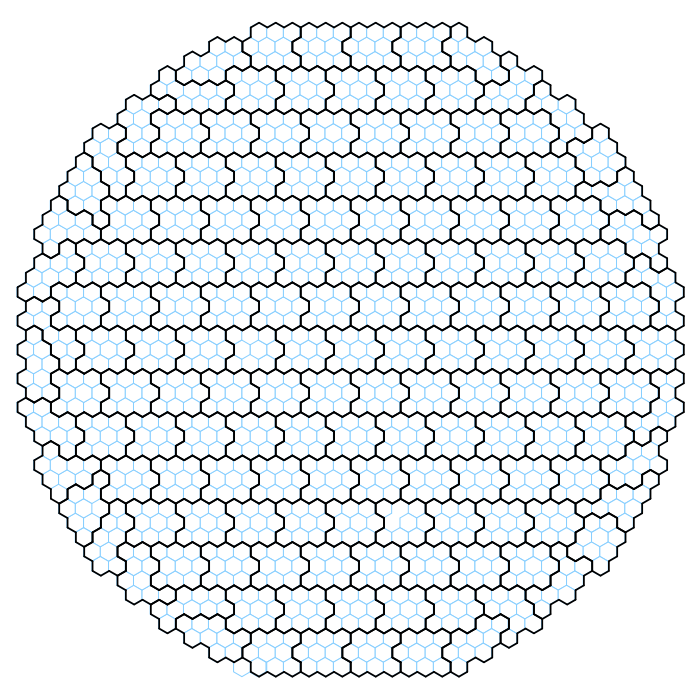

Figure 3: Trigger and readout patches of the FACT camera. Each patch consists of 9 pixels. can make any single photo-electron (pe) to produce a rather large signal. As a countermeasure to one pixel triggering the full camera, sum-trigger implementations often apply clamping, i.e. they limit the maximum contribution of each single pixel by cutting their signal at a fixed amplitude. Implementing this in analog electronics requires quite an effort for each pixel. In addition, FACT needs to be operated at very different conditions of ambient light: during dark nights the measured pe rate per pixel is $\approx 30 \mathrm{MHz}$, while FACT routinely operates also at rates exceeding one $\mathrm{GHz}$ per pixel due to moonlight. Therefore, not only the trigger threshold per patch but also the clamping amplitude per pixel would need to be programmable. In FACT, we operate the G-APDs at a moderate crosstalk probability of $\approx 12 \%$. Taking correctly into account multiple crosstalk, one can estimate that even during bright moonlight conditions less than one photon per second would produce a 20 pe signal anywhere in the camera [2], a level significantly lower than a reasonable trigger threshold even during dark nights. A more detailed analysis has to take into account pile-up of other signals in the same patch and contamination by bright stars. From this we concluded that accidental single pixel triggers to be rare and clamping not to be necessary. Analyzing the events written to disk and applying a simulated clamping level at 50\% of the applied trigger threshold shows that the trigger rate would be reduced by only $\approx 2 \%$, confirming that electronic clamping is not necessary for sum-triggers when using G-APDs at moderate crosstalk levels. A study about the effect of different crosstalk values is presented in [17].

\section{Performance and Longterm Stability}

One feature of G-APDs is their strong dependency on temperature. Therefore, we initially implemented a calibration system using a stabilized light-pulser that can be triggered to illuminate the camera with very short pulses. It is not sufficient to apply a calibration to the signals at the analysis step, since e.g. a modified gain affects the trigger threshold. Therefore, a feedback system 
to adjust the voltage applied to the G-APDs in real time is included. After few months of operation it became evident that the external calibration system is not necessary. It is sufficient to measure the temperature close to the sensors and adjust the voltage accordingly. It was not foreseen to measure the voltage directly at the sensors, but only at the power supply. Due to voltage drops on serial resistors, one has also to take the DC current into account to adjust the voltage. A summary of this system and the performance reached can be found in [18] and more detailed in [2]. It was also realized that the measurement of the voltage at the power supply (situated in a container next to the telescope) has a temperature dependency. So it is necessary to keep the container at a rather constant temperature to further improve the stability. An improved power supply overcoming this problem is presented in [19]. For future cameras, it would be beneficial to measure temperatures as well as the applied voltage directly at each sensor.

Another calibration method is based on single-pe spectra: due to dark-noise and optical crosstalk, a single-pe spectrum can be taken for each pixel even when the lid of the camera is closed. Comparing the signals for $\mathrm{n}$ and $\mathrm{n}+1$ pe is a measurement of the gain of each pixel. This allows to crosscheck the temperature correction and the longterm stability as well as to flatfield the gain over the full camera. A parameterization up to 8 pe is implemented, limited by low statistics for even higher signals $[2,18]$. It has to be noted that when applying to each chip the voltage given on their individual datasheet from Hamamatsu, the measured gain difference is less than $2.1 \%$, corresponding to $\approx 30 \mathrm{mV}$ and comparable to the resolution of the power supply used.

An additional method is based on trigger-ratescans (fig.4). At low thresholds, the rate is due to accidental triggers induced by ambient light photons. But at high thresholds, the rate is dominated by Cherenkov showers induced by cosmic ray particles. For a given orientation of the telescope the rate of cosmic ray particles is constant, so comparing measured trigger rates allows us to prove that when operating the feedback system, the performance of the sensors is not affected by the amount of ambient light $[2,18]$. In addition, these ratescans can be used to identify bad atmospheric conditions [20,2].

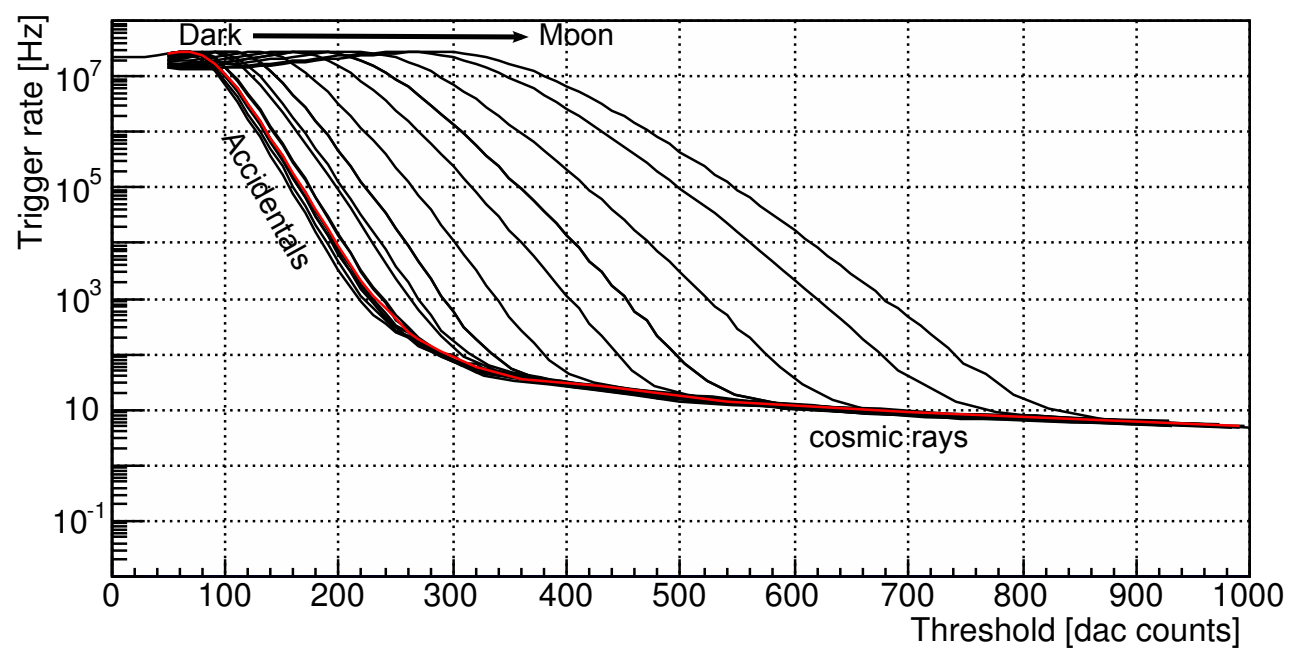

Figure 4: Ratescans taken with good weather but different temperatures and moonlight conditions in the time frame Jan. - June 2012 (black) [20] and June 2015 (red). There is no indication for any dependency of the cosmic ray trigger rate on temperature, ambient light level or age of the sensors. 
Last but not least, muon ring images can be used for calibration [22]. They allow to check the optical quality of the telescope and the time resolution of the system.

A very important question is the longterm stability of this novel sensor type. In the past 3.5 years of operation, FACT has taken data during more than 5000 hours. While during dark nights, the DC current per pixel is $\approx 5 \mu \mathrm{A}$, FACT also takes regularly data with DC currents up to $\approx 100 \mu \mathrm{A}$ (fig.5). Integrating the actual DC currents over time and dividing by the DC current corresponding to dark night, each pixel in the FACT camera has so far accumulated a charge corresponding to more than 14000 hours of operation under moonless conditions. The dark current is $\approx 0.5 \mu \mathrm{A}$ (and a rate $\approx 3 \mathrm{MHz}$ ) per pixel. So the total charge accumulated by each pixel corresponds to more than 140000 hours $(\approx 16$ years continuous operation) under dark laboratory conditions.

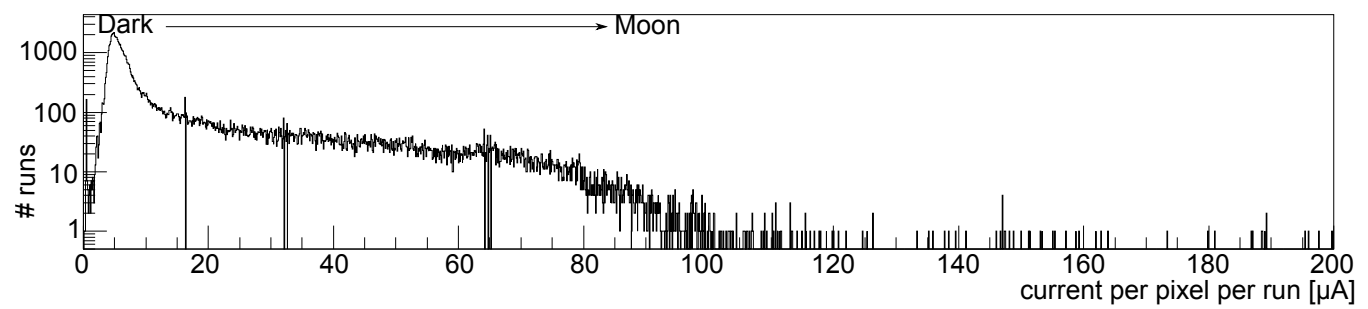

Figure 5: Average DC current per pixel and run. Standard data runs have a duration of five minutes each (except close to twilight when runs are shortened to one minute to avoid too strong changes within one run). Special runs regularly taken to monitor the stability of the system have a duration of 40 seconds.

All pixels are still operated under the exactly same applied voltages as three years ago, and neither of the mentioned calibration methods shows any hint of aging effects at a level of a few percent per year (fig.4). While readjusting the mirrors using a novel alignment method [21] improved the optical quality of the system [22], it has a very small effect on the trigger rates because the majority of photons still end up in the same trigger patch.

Taking into account that we operate more than 1400 sensors in parallel, it can safely be concluded that our G-APDs show an excellent longterm behavior. Nevertheless, we are currently working on improved analysis methods to be able to look for even smaller effects.

One pixel recently stopped delivering a signal. This happened in coincidence with a period of very bad weather during winter 2014/15 that resulted in a humidity problem inside the camera. Investigating the damage in the camera on-site hints towards electro-corrosion of a hidden connector. But without complete disassembly of the camera, it cannot be excluded that the sensor itself died for some reason. Nevertheless, this would at worst be one out of 1440 sensors dying within 3.5 years of continuous operation under very harsh conditions, corresponding to a failure rate of less than $0.02 \%$ per year.

\section{Status and Outlook}

FACT has now operated for more than 3.5 years under harsh conditions, most of the time from remote without the need of a data taking crew on-site. This reduces significantly the operation costs. The excellent longterm stability of the system and the possibility to operate under changing moonlight condition makes it ideal for longterm monitoring of variable sources [3], and the measured spectrum from the Crab nebula is consistent with the one measured by other IACTs [23]. 
The performance, reliability and stability of FACT clearly proves that G-APDs are a viable alternative for future IACTs as well as other detectors, and several systems are currently under development [24]. G-APDs are not a one-to-one replacement for PMTs. An initially unexpected benefit of G-APDs is that by methods developed by us, a camera can be made self-calibrating without the need of any external calibration device (that itself would need its own proof of longterm stability).

Currently, G-APDs are limited to rather small sizes compared to areas that can be covered with PMTs. Nevertheless, already the operation of Module 0 as well as the sum-trigger system of FACT clearly show that it is easy to sum several individual sensors to form a a larger pixel with common readout channel.

It has also to be noted that the sensors used in FACT belong to the first generation of GAPDs available in large quantities. Recently, better sensors with improvements in photodetection efficiency, crosstalk, afterpulsing and dark noise were developed by several vendors. In addition, sensors better adjusted to the wavelength range of Cherenkov showers (and less sensitive to ambient light at longer wavelengths) became available. This will significantly increase the amount of Cherenkov photons collected per event and therefore lower the energy threshold of a comparable system. Registering less ambient light photons will improve the operation and data quality of observations during bright nights. Therefore, one can expect that upcoming IACTs based on SiPM (aka G-APDs) will perform even better than FACT does.

\section{Acknowledgment}

The important contributions from ETH Zurich grants ETH-10.08-2 and ETH-27.12-1 as well as the funding by the German BMBF (Verbundforschung Astro- und Astroteilchenphysik) and HAP (Helmoltz Alliance for Astroparticle Physics) are gratefully acknowledged. We are thankful for the very valuable contributions from E. Lorenz, D. Renker and G. Viertel during the early phase of the project. We thank the Instituto de Astrofisica de Canarias allowing us to operate the telescope at the Observatorio del Roque de los Muchachos in La Palma, the Max-Planck-Institut für Physik for providing us with the mount of the former HEGRA CT 3 telescope, and the MAGIC collaboration for their support. Part of this work is supported by Deutsche Forschungsgemeinschaft (DFG) within the Collaborative Research Center SFB 876 "Providing Information by Research-Constrained Analysis", project C3.

\section{References}

[1] H. Anderhub et al. (FACT Collaboration), Design and operation of FACT - the first G-APD Cherenkov telescope, JINST 8 P06008, 2013

[2] A. Biland et al. (FACT Collaboration), Calibration and performance of the photon sensor response of FACT - the first G-APD Cherenkov telescope, JINST 9 P10012, 2014

[3] D. Dorner at al. (FACT Collaboration), FACT - TeV Flare Alerts Triggering Multi-Wavelength Observations, In: Proceedings of the 34th ICRC, 704, The Hague, 2015 (these proceedings)

[4] T. Bretz et al., MARS - Cheops ed., A flexible software framework for future Cherenkov telescopes, In: proceedings of the 11th ICATPP Conference, 2009 
[5] D. Dorner et al. (FACT Collaboration), FACT - Monitoring Blazars at Very High Energies, In: Proceedings of the Fermi Symposium, 2014

[6] K.A. Brügge et al. (FACT Collaboration), FACT-Tools - Streamed Real-Time Analysis, In: Proceedings of the 34th ICRC, 865, The Hague, 2015 (these proceedings)

[7] D. Renker and E. Lorenz, Advances in solid state photon detectors, JINST 4 P04004, 2009

[8] A. Biland et al., First detection of Cherenkov light from cosmic-particle-induced air showers by Geiger-mode avalanche photodiodes, Nucl. Instr. and Meth. A 581 143-146, 2007

[9] M. Actis et al. Design Concepts for the Cherenkov Telescope Array CTA, An Advanced Facility for Ground-Based High-Energy Gamma-Ray Astronomy, Exp. Astronomy, 32.3 193-316, 2011

[10] I. Braun et al. (FACT Collaboration), First Avalanche-photodiode camera test (FACT): A novel camera using G-APDs for the observation of very high-energy $\gamma$-rays with Cherenkov telescopes, Nucl. Instr. and Meth. A 610 400-403, 2009

[11] H. Anderhub et al. (FACT Collaboration), A novel camera type for very high energy gamma-ray astronomy based on Geiger-mode avalanche photodiodes, JINST 4 P10010, 2009

[12] S. Ritt et al., Application of the DRS chip for fast waveform digitizing; Nucl. Instr. and Meth. A 623 486, 2010

[13] Hamamatsu Photonics K.K., MPPC data sheet, 2008

[14] H. Anderhub et al. (FACT Collaboration), Electronics for the camera of the First G-APD Cherenkov Telescope (FACT) for ground based gamma-ray astronomy, JINST 7 C01073, 2012

[15] T. Bretz et al. (FACT Collaboration), FACT - The first G-APD Cherenkov telescope (first results), In: AIP Conf. Proc. 1505 773-776, 2012

[16] M. Rissi et al. A New Sum Trigger to Provide a Lower Energy Threshold for the MAGIC Telescope, IEEE Transactions on Nuclear Science, 56 6, 2009

[17] J. Buss et al. (FACT Collaboration), FACT - Influence of SiPM Crosstalk on the Performance of an Operating Cherenkov Telescope, In: Proceedings of the 34th ICRC, 863, The Hague, 2015 (these proceedings)

[18] D. Neise et al. (FACT Collaboration), FACT - Performance of the First SiPM camera, In: Proceedings of the 34th ICRC, 706, The Hague, 2015 (these proceedings)

[19] J. Schumacher et al. Dedicated power supply system for silicon photomultipliers, In: Proceedings of the 34th ICRC, The Hague, 2015 (these proceedings)

[20] D. Hildebrand et al. (FACT Collaboration), FACT - Measuring Atmospheric Conditions with Imaging Air Cherenkov Telescopes, In: Proceedings of the 33rd ICRC, Rio de Janeiro, 2013

[21] S. Müller et al. (FACT Collaboration), FACT - Novel mirror alignment using Bokeh and enhancement of the VERITAS SCCAN alignment method, In: Proceedings of the 34th ICRC, 976, The Hague, 2015 (these proceedings)

[22] M. Nöthe et al. (FACT Collaboration), FACT - Calibration of Imaging Atmospheric Cherenkov Telescopes with Muon Rings, In: Proceedings of the 34th ICRC, 733, The Hague, 2015 (these proceedings)

[23] F. Temme et al. (FACT Collaboration), FACT - First Energy Spectrum from a SiPM Cherenkov Telescope, In: Proceedings of the 34th ICRC, 707, The Hague, 2015 (these proceedings)

[24] Several projects presented in: Proceedings of the 34th ICRC, The Hague, 2015 (these proceedings) 